\title{
Get Aroused and Be Stronger: Emotional Facilitation of Physical Effort in the Human Brain
}

\author{
Liane Schmidt, ${ }^{1,5}$ Marie-Laure Cléry-Melin, ${ }^{1,5}$ Gilles Lafargue, ${ }^{6,7}$ Romain Valabrègue, ${ }^{1,2,5}$ Philippe Fossati, ${ }^{3,4,5}$ \\ Bruno Dubois, ${ }^{1,4,5}$ and Mathias Pessiglione ${ }^{1,2,5}$ \\ ${ }^{1}$ Institut du Cerveau et de la Moëlle épinière (ICM), INSERM UMRS 975, ${ }^{2}$ Centre de NeuroImagerie de Recherche (CENIR), ${ }^{3}$ CNRS UMR 7593, and ${ }^{4} \mathrm{Hôpital}$ \\ Pitié-Salpêtrière, Assistance Publique-Hôpitaux de Paris, 75013 Paris, France, ${ }^{5}$ Université Pierre et Marie Curie (Paris 6), 75005 Paris, France, ${ }^{6} \mathrm{CNRS}$ UMR \\ 8160, 59037 Lille, France, and ${ }^{7}$ Université Lille Nord-de-France (Lille 3), 59653 Villeneuve d'Asq, France
}

Effort magnitude is commonly thought to reflect motivation, but little is known about the influence of emotional factors. Here, we manipulated the emotional state of subjects, via the presentation of pictures, before they exerted physical effort to win money. After highly arousing pictures, subjects produced more force and reported lower effort sensation, regardless of monetary incentives. Functional neuroimaging revealed that emotional arousal, as indexed by postscan ratings, specifically correlated with bilateral activity in the ventrolateral prefrontal cortex. We suggest that this region, by driving the motor cortex, constitutes a brain pathway that allows emotional arousal to facilitate physical effort.

\section{Introduction}

In sports that crucially depend on energizing muscle, such as weightlifting, records are often broken during prestigious competitions like the Olympic Games. This may be attributable to incentive motivation, since expected rewards, in terms of fame or money, are higher during such athletic events than during smaller-scale competitions. Another reason may be that emotional arousal, elicited by the presence of cameras and large audiences, boosts maximal force. This hypothesis was first suggested a long time ago in sport psychology but has remained virtually unexplored (Tod et al., 2003), despite the large body of literature showing the positive impact of emotional arousal on various processes such as attention, perception, and memory (Hamann et al., 1999; Anderson and Phelps, 2001; Ohman et al., 2001; Dolcos et al., 2005; Anderson et al., 2006). In the case of maximal force, a boosting effect is not necessarily expected, however, since emotional arousal has been shown to impair motor control (Noteboom et al., 2001; Coombes et al., 2008). Furthermore, the effect might depend on the valence of the emotional state, as reported for both motor and cognitive control (Gray et al., 2002; Coombes et al., 2007), meaning that performance would be enhanced in supportive environments but deteriorated in adverse ones. The aim of this study was to test the influence of both positive and negative emotional arousal on force produc-

\footnotetext{
Received April 24, 2009; accepted May 27, 2009

This study was funded by the Fyssen Foundation. L.S. received a fellowship from the French Ministère de la Recherche. We thank Chris Frith for his helpful suggestions on an earlier version of this manuscript. We are also grateful to Eric Featherstone for designing the MRI-compatible grippers, to the Centre de Neurolmagerie de Recherche staff (Eric Bardinet, Eric Bertasi, Kevin Nigaud) for their skilful assistance in MRI data acquisition and analysis, and to Jean-Claude Dreher for providing the skin conductance recording device. Guillaume Marrelec and Hilke Plassmann gave precious advice about data analysis. Soledad Jorge and Shadia Kawa checked the English.

Correspondence should be addressed to Mathias Pessiglione at the above address. E-mail: mathias.pessiglione@gmail.com.

DOI:10.1523/JNEUROSCI.1951-09.2009

Copyright $\odot 2009$ Society for Neuroscience $\quad$ 0270-6474/09/299450-08\$15.00/0
}

tion, to dissociate these effects from incentive motivation, and to identify the underlying neural correlates.

To this end, we adapted our incentive force task, in which subjects must squeeze a hand grip to win money (Pessiglione et al., 2007; Schmidt et al., 2008). The amount of money at stake $(0.01,0.1$, or $1 €)$ was randomly varied on a trial-by-trial basis, and subjects were told that they would be allowed to keep a fraction of that monetary incentive, corresponding to the force produced. Subjects were instructed to initiate force production when the monetary incentives and a thermometer simultaneously appeared on the screen. Real-time feedback about the force exerted was given by way of fluctuating fluid levels within the thermometer, and feedback on the cumulative amount earned was presented at the end of every trial (see Fig. 1). We introduced to this paradigm images from the International Affective Picture System (IAPS) (Lang et al., 2005), which could be emotionally negative, neutral, or positive. Different emotional pictures were randomly selected and displayed on every trial before force production. Subjects were told that pictures would not affect monetary payoff, so the effect of our emotional manipulation on grip force could be regarded as implicit. The present paradigm thus makes it possible to disentangle the effects of emotion (induced by pictures) from those of motivation (induced by incentives).

\section{Materials and Methods}

This study was approved by the Pitié-Salpêtrière Hospital ethics committee. Participants were recruited via e-mail and gave informed consent before participating. We start below with the description of the procedures used for the functional magnetic resonance imaging (fMRI) study, which was conducted on 20 subjects. Two other groups of 20 subjects performed the behavioral task in the pilot studies, with slight variations in the procedures that are detailed in the next paragraphs.

Behavioral data acquisition (fMRI study). A total of 20 subjects (aged $24 \pm 1$ years, 10/10 males/females, all right-handed) were scanned. Participants were screened for the following exclusion criteria: $<18$ years of age, currently taking drugs or medications, history of psychiatric or neu- 


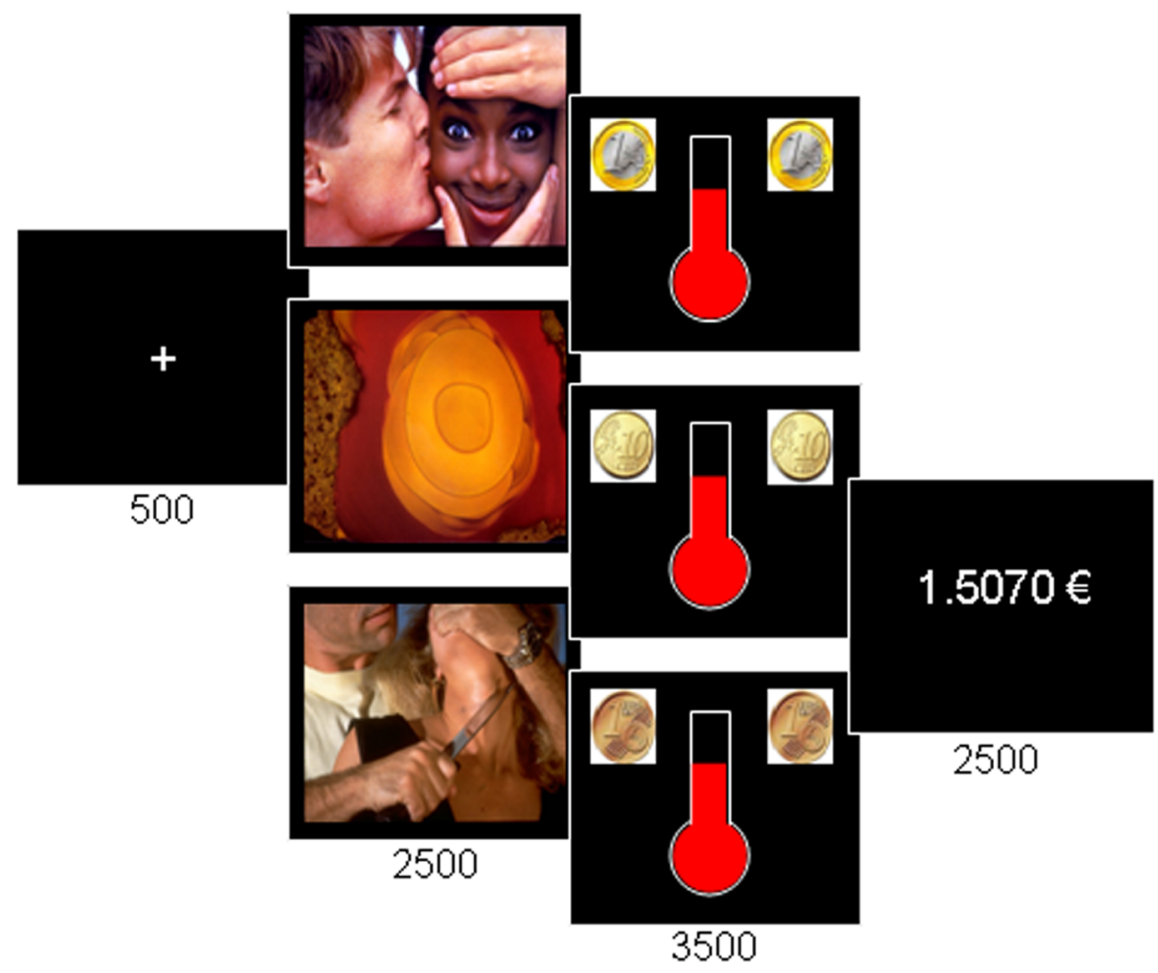

Figure 1. The emotional incentive force task. Successive screens displayed in every trial are shown from left to right, with durations in milliseconds. Emotional pictures that could be neutral or arousing (with positive or negative valence) were shown before physical effort. Effort was triggered by simultaneously showing the amount of money at stake, materialized as coin images ( 1 cent, 10 cents, or $1 €$ ), and a thermometer in which fluid level represented the force exerted on the hand grip. Subjects knew that the top of the thermometer corresponded to the monetary incentive, such that the more they squeezed the hand grip, the more money they would win. The last screen informed subjects about the cumulative total of monetary earnings.

rological illness, left-handedness and contraindications to MRI scanning (pregnancy, claustrophobia, metallic implants). They believed that they would be playing for real money, but to avoid discrimination, payoff was rounded up to a fixed amount of $100 €$ for every participant.

Before scanning, subjects were given instructions for the task and were allowed to perform a short practice version (27 trials), to become familiarized with stimulus presentation and hand grip manipulation. Subsequently, they were escorted inside the scanner and were invited to find an optimal body position, while lying down with the power grip in their right hand, the arm resting over the belly. The power grip was made up of two molded plastic cylinders which compressed an air tube when squeezed (provided by the Wellcome Trust Centre for Neuroimaging, London, UK). The tube led to the control room, where it was connected to a transducer capable of converting air pressure into voltage. Thus, compression of the two cylinders by an isometric handgrip resulted in the generation of a differential voltage signal, linearly proportional to the force exerted. The signal was fed to the stimuli presentation personal computer (PC) via a signal conditioner (CED 1401; Cambridge Electronic Design). Stimulus presentation was programmed with Cogent 2000 (Wellcome Department of Imaging Neuroscience, London, UK). The visual stimuli were displayed behind the scanner on a projector screen, which subjects could see via mirrors positioned over their eyes. The dynamic changes in recorded signal were used to provide subjects with real-time visual feedback on the force being exerted on the grip, which appeared as a fluid moving up and down within a thermometer (Fig. 1). We calibrated the baseline ("do nothing") and measured the maximal force ("squeeze the grip as hard as you can") before starting the experiment.

During the experiment, galvanic skin conductance level was continuously monitored via two electrodes placed on dorsal and ventral palmar surfaces of the left hand. Electrodes were connected to a skin conductance processing unit (Student Lab Pro 3.7.0; Biopac Systems) in the control room. The analog signal was displayed online on a second PC screen and digitalized using a sampling frequency of $5000 \mathrm{~Hz}$. The digital signal was lowpass filtered using the Matlab signal processing toolbox (Matlab R2006b; The MathWorks) and down-sampled to a frequency of $200 \mathrm{~Hz}$.

Subjects performed three sessions of the emotional incentive force task. Each session lasted $\sim 12$ min and included nine repetitions of nine trial types, for a total of 81 trials. The nine trial types corresponded to a $3^{\star} 3$ factorial design: three monetary incentives $(0.01,0.1$, or $1 €$ ) three emotional categories (negative, neutral, or positive). Monetary incentives and emotional categories were randomly distributed over the series of trials. In addition, we varied the height (by scaling it to 50,75 , or $100 \%$ of the thermometer length) that subjects could reach by producing their maximal force, which was measured beforehand, to decorrelate spatial location of fluid level (and hence monetary earning) from the variables of interest. In every trial, subjects had to fixate attention on a central cross and then watch a new picture that was randomly selected from the a priori emotional categories and displayed on screen for $2500 \mathrm{~ms}$. Subjects were told that the content of these pictures would not matter in the calculation of monetary payoff. The thermometer then appeared on the screen, together with coin images indicating the amount of money at stake. This was the trigger signal for subjects to squeeze the hand grip and attempt to move the fluid level up as high as possible, within a $3500 \mathrm{~ms}$ interval. Subjects were aware that the height they reached within the thermometer determined the fraction of the monetary stake they would keep. At the end of every trial, a cumulative total was displayed for $2500 \mathrm{~ms}$, indicating the amount of money won so far. Random time intervals (jitters of $\pm 500 \mathrm{~ms}$ ) were inserted into every trial to ensure better sampling of the hemodynamic response and to avoid the sleepiness that can result from a monotonous pace.

Emotional pictures were chosen based on valence and arousal ratings provided by the IAPS (Lang et al., 2005). Categories were derived from the valence ratings, with ranges of 1.51-3.94 for negative, $4.30-5.88$ for neutral, and $6.29-8.34$ for positive pictures. We controlled for luminance and dimension between pictures and balanced the proportion of social and nonsocial pictures between categories. For categorical comparisons, we kept the a priori categories that served to balance the design. As a further control, we asked subjects to rate the valence and arousal of the pictures used in the emotional incentive force task. These a posteriori ratings, which were very similar to the a priori ratings provided by the IAPS, were used in all parametric analyses. The control rating task was performed after functional neuroimaging, during acquisition of the structural scan. Each picture was displayed again for $3 \mathrm{~s}$ after a fixation cross, and subjects had to move a cursor along an analog scale graduated from -10 (maximal negative arousal) to +10 (maximal positive arousal), with 0 standing for minimal arousal. To move the cursor left and right, subjects pressed buttons on a keypad with their index or middle finger. There was no time limit to respond; subjects moved on to the next trial at their own pace by pressing a specified button of the keypad. Each of the 243 pictures (which had been divided into three sessions of 81 trials in the emotional incentive force task) was displayed once, for a total duration of $\sim 20 \mathrm{~min}$, on average.

Behavioral data acquisition (pilot studies). A total of 40 subjects (aged $25 \pm 2$ with 18/22 males/females and 34/6 right-/left-handed) participated in the behavioral studies. Participants were screened for the following exclusion criteria: $<18$ years of age, currently taking drugs or medications, and history of psychiatric or neurological illness. They were 
informed that they would not be paid for their participation and that incentives used for behavioral assessment would be fictive.

The material used for the behavioral studies was different from that used for the fMRI study. Stimulus presentation was programmed on a PC using Paradigmae software (Paradigmae, e(ye)BRAIN, Paris, France; www.eye-brain.com). Force was recorded using a "pinch grip" (MIE Medical Research) with a sample rate of $25 \mathrm{~Hz}$. Skin conductance was recorded using $\mathrm{Ag} / \mathrm{AgCl}$ electrodes ( $1 \mathrm{~cm}$ diameter) taped onto the palmar surface of the midfinger and forearm of the nondominant hand. The signal was fed to a skin conductance processing unit (Psylab SC5 Stand Alone Monitor System; Contact Precision Instruments). The filtered analog of the skin conductance was displayed online and recorded digitally, with a sample rate of $200 \mathrm{~Hz}$, on a supplementary PC that received event markers from the PC running stimuli presentation and recording grip force.

Task structure was similar to that of the fMRI experiment, with slight variations in the timing, as well as some important differences. The first behavioral study (20 subjects) consisted of 15 repetitions of six trial types, for a total of 90 trials grouped into a single session lasting $13.5 \mathrm{~min}$. The six trial types corresponded to a $2 \star 3$ factorial design, with two monetary incentives $(0.01$ and $1 €$ ) and three emotional categories (negative, neutral, and positive). The second behavioral study (20 subjects) was made up of 12 repetitions of nine trial types, for a total of 108 trials, again grouped into a single session lasting $19.8 \mathrm{~min}$. The nine trial types were generated according to the three monetary incentives $(0.01,0.1$, and $1 €)$ times the three emotional categories (positive, neutral, negative). In comparison with the first behavioral study, we introduced an intermediate incentive $(0.1 €)$ to assess linear modulation instead of all-or-nothing effects. In this study, subjects were asked to rate the effort exerted when squeezing the hand grip. The precise question was "how hard did you try?" and subjects were encouraged to report their feeling and not to rely on visual feedback (fluid level). Rating was performed after the thermometer screen but before showing the monetary outcome. To deliver their rating, subjects had to move a cursor along an analog scale graduated from 0 (minimal effort) to 10 (maximal effort). They used the keyboard to move the cursor left and right and had $3 \mathrm{~s}$ to reach the appropriate position. To prevent subjects from reporting visual feedback and not subjective feeling, we varied the height (scaled to 60,70 , and $80 \%$ of the thermometer length) they would reach when producing their maximal force. The main features of this second behavioral design were retained in the fMRI experiment, with the exception of effort ratings, which were skipped to reduce scanning time.

Behavioral data analysis. Several dependent variables were considered: grip force, skin conductance, and subjective ratings (arousal, valence, and effort). Grip force was extracted both as the maximum reached and the area under the curve. We retained the latter for the results described in the main text, but they were all significant for both measures. Skin conductance was extracted as the difference between the peak and a minimum taken before thermometer display onset. Grip force, skin conductance, and effort rating were expressed as a percentage of the highest measure. Effort ratings were divided by the observed grip force, on a trial-by-trial basis. Although we kept raw percentages and ratings for illustration purposes, all dependent variables were converted into $z$-scores for statistical analysis. A global ANOVA was first conducted to test main effects of group (first behavioral, second behavioral, fMRI studies), incentive (0.01€ vs $1 €$ ) and picture (arousing or neutral), as well as interactions. We found no significant effect of group, significant effects of arousal $\left(F_{(1,58)}=5.51, p<0.05\right)$ and motivation $\left(F_{(1,58)}=552.07, p<\right.$ 0.001 ), and no significant interaction between arousal and group or motivation. We, therefore, pooled the 60 subjects together and considered emotional and motivational factors separately. Post hoc comparisons between incentive and emotional categories were then performed using one-tailed paired $t$ tests. One-tailed $t$ tests across subjects were also used to test the significance of the coefficients obtained from multiple regressions of grip force against arousal, valence, and incentive. Three statistical significance thresholds were considered: $p<0.05, p<0.01$, and $p<$ 0.001 . All statistical tests, including regressions, were conducted with the Matlab Statistical Toolbox (Matlab R2006b; The MathWorks).
Table 1. Main activations at the time of physical effort

\begin{tabular}{llllll}
\hline Brain region & Side & $\begin{array}{l}\text { Number } \\
\text { of voxels }\end{array}$ & $t$ value & Zscore & $\begin{array}{l}\text { MNI coordinates } \\
(x, y, z ; m m)\end{array}$ \\
\hline $\begin{array}{l}\text { Arousal } \\
\quad \text { VLPFC }\end{array}$ & $\mathrm{R}$ & 554 & 5.29 & 4.10 & $50,30,-14$ \\
$\quad$ VLPFC & $\mathrm{L}$ & 129 & 5.22 & 4.06 & $-56,30,-4$ \\
$\quad$ Insula & $\mathrm{L}$ & & 4.96 & 3.92 & $-52,16,-12$ \\
$\begin{array}{l}\text { Incentive } \\
\text { Basal forebrain }\end{array}$ & $\mathrm{L}$ & 220 & 6.49 & 4.66 & $-14,2,-2$ \\
$\quad$ Basal forebrain & $\mathrm{R}$ & & 4.29 & 3.54 & $8,-4,-2$ \\
$\quad$ Insula & $\mathrm{L}$ & 101 & 5.67 & 4.28 & $-66,-10,-2$ \\
Insula & $\mathrm{R}$ & 949 & 4.96 & 3.93 & $28,16,-28$ \\
$\quad$ Cerebellum & $\mathrm{R}$ & 646 & 5.88 & 4.39 & $20,-90,-24$ \\
M1 & $\mathrm{L}$ & 424 & 5.74 & 4.32 & $-18,-30,78$ \\
$\quad$ SMA & $\mathrm{L}$ & & 5.43 & 4.17 & $2,-38,74$ \\
Force & & & & & \\
$\quad$ M1 & $\mathrm{L}$ & 295 & 6.59 & 4.70 & $-34,-22,64$ \\
Insula & $\mathrm{R}$ & 378 & 5.49 & 4.20 & $56,22,-8$ \\
$\quad$ Insula & $\mathrm{L}$ & 146 & 5.08 & 3.99 & $-68,-14,-4$ \\
$\quad$ Cerebellum & $\mathrm{R}$ & 186 & 4.67 & 3.76 & $36,-40,-42$ \\
$\quad$ Cerebellum & $\mathrm{L}$ & 113 & 4.24 & 3.51 & $-16,-82,-50$ \\
\hline
\end{tabular}

The listed clusters included a minimum of 100 voxels and showed significant activation $(p<0.001$, uncorrected) in relation to arousal ratings, monetary incentives, and forces produced across trials. SMA, Supplementary motor area; $R$, right; $L$, left.

Imaging data acquisition. T2*-weighted echo planar images (EPI) were acquired with blood oxygen level-dependant (BOLD) contrast on a 3.0 Tesla magnetic resonance scanner (Siemens Trio). A tilted plane acquisition sequence was used to optimize functional sensitivity in the orbitofrontal cortex. To cover the whole brain with a repetition time of $1.98 \mathrm{~s}$, we used the following parameters: 30 slices; $2 \mathrm{~mm}$ slice thickness; $2 \mathrm{~mm}$ interslice gap. T1-weighted structural images were also acquired, coregistered with the mean EPI, segmented and normalized to a standard T1 template, and averaged across all subjects to allow group-level anatomical localization. EPI images were analyzed in an event-related manner, within a general linear model, using the statistical parametric mapping (SPM) software SPM5 (Wellcome Department of Imaging Neuroscience). The first five volumes of each session were discarded, to allow for T1 equilibration effects. Preprocessing consisted of spatial realignment, normalization using the same transformation as structural images, and spatial smoothing using a Gaussian kernel with a full-width at halfmaximum of $8 \mathrm{~mm}$.

Imaging data analysis. We used a single linear regression model to generate all SPMs as follows. Each trial was modeled as having three time points, corresponding to picture, thermometer, and outcome display onsets. We then included parameters measured from or imposed to the subjects who participated in the fMRI study. The design matrix contained six parametric modulators, arousal ratings at the time of picture display, arousal ratings, monetary incentives, recorded force and fluid level scaling at the time of thermometer display, and monetary earning (for each particular trial) at the time of outcome display. Thus, the design matrix contained nine regressors of interest (three stick functions and six parametric modulations orthogonalized from left to right), all convolved with a canonical hemodynamic response function (HRF). To correct for motion artifacts, subject-specific realignment parameters were modeled as covariates of no interest. Linear contrasts of regression coefficients were computed at the individual subject level and then taken to a grouplevel random effect analysis (one-sample $t$ test). All reported significant activations (Table 1) contained a minimum of 100 voxels, which corresponded to a threshold of $p<0.05$ after correction for multiple comparisons at the cluster level.

To extract regression coefficients in maxima of interest [ventrolateral prefrontal cortex (VLPFC), basal forebrain (BF), and primary motor cortex (M1)], we used a second linear regression model. The nine conditions (three monetary incentives times three emotional categories) were modeled as separate regressors that contained $6 \mathrm{~s}$ boxcars encompassing picture display and effort production. Boxcars of $2.5 \mathrm{~s}$ were also added to model the feedback periods, giving a total of 10 regressors of 
interest convolved with the canonical HRF. Regression coefficients were extracted from the SPM global maxima at the individual level for statistical comparisons (one-tailed paired $t$ tests) based on intersubject variance.

Finally, we examined whether significant activations in VLPFC and BF would explain M1 activation. A third linear regression model was built to this aim, with all trials containing a single $6 \mathrm{~s}$ boxcar, covering picture display plus effort period, and modeled as separate regressors. Thus, the design matrix contained 243 regressors of interest, each containing one boxcar convolved with the canonical HRF, plus the realignment parameters. In this way, we obtained an estimation of the hemodynamic response magnitude for every single trial. These response magnitudes were then extracted for the different maxima of interest and taken to multiple regressions of M1 against VLPFC and BF activations. Significance of regression coefficients was tested using one-sample $t$ tests across subjects.

To estimate BOLD time courses, the raw signal was extracted from each global maximum, resampled using interpolation between scans, high-pass filtered, cut at picture display onset, and averaged across trials, sessions, and subjects.

\section{Results}

Both categorical analysis (comparisons between arousal or incentive levels) and parametric analysis (correlations with arousal or incentive levels) were conducted to assess the effects of emotional and motivational manipulations. Regarding emotion, we kept the a priori categories derived from the IAPS for categorical comparisons but used the ratings obtained from our subjects (Fig. 2A) for all parametric analyses. As will be shown below, the categorical and parametric analyses converged to the same conclusions.

\section{Behavioral data}

The main dependent variable was the amount of force produced during each trial (Fig. $2 B$, top). A previous finding that grip force increases with monetary incentives was replicated, with significant paired $t$ tests for all comparisons: 0.01 vs $0.1 €\left(t_{(39)}=7.8\right.$, $p<0.001), 0.1$ vs $1 €\left(t_{(39)}=11.8, p<0.001\right)$. Crucially, subjects produced more force after negative or positive pictures relative to neutral ones $\left(t_{(59)}=4.1, p<0.001\right.$ and $\left.t_{(59)}=3.2, p<0.01\right)$ but did not significantly differentiate between positive and negative pictures. We found no significant interaction between arousal and motivation: emotional pictures had the same impact on force production whatever the reward at stake.

To further assess the influence of the different factors on grip force, we conducted a linear regression across trials for each subject, with arousal, valence, and incentive as explanatory variables and force as the variable to be explained (see Fig. $4 A$ ). Note that the three explanatory factors were orthogonal in our experimental design. Regression coefficients were statistically significant across subjects for incentive $\left(r=0.4, t_{(59)}=12.2, p<0.001\right)$ and arousal $\left(r=0.06, t_{(59)}=3.7, p<0.001\right)$ but not for valence nor for the interactions.

Skin conductance response was triggered at the time of force production, starting $\sim 3 \mathrm{~s}$ and peaking $\sim 5 \mathrm{~s}$ after thermometer display onset (see Fig. 4A). Similar results were found (Fig. $2 B$, middle): significant increases with positive and negative pictures relative to neutral ones $\left(t_{(59)}=1.9, p<0.05 ; t_{(59)}=2.9, p<0.01\right)$ but no significant difference between positive and negative pictures. Considering monetary incentives, we also found a significant difference between the $1 €$ condition and the two others $\left(t_{(59)}=6.7, p<\right.$ 0.001 and $\left.t_{(59)}=7.3, p<0.001\right)$. Thus, as seen with grip force, the relevant factors that appeared to influence skin conductance were emotional arousal (but not valence) and monetary incentives.

Subjective judgments about effort intensity ("how hard did you try?") were examined after scaling proportional to the objec-
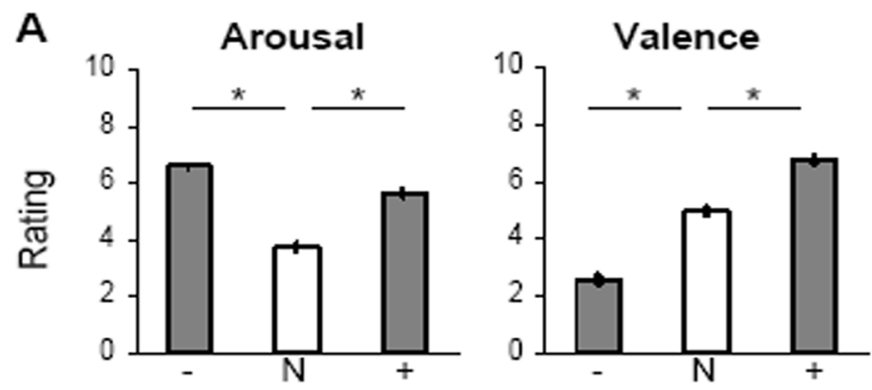

B

Emotion
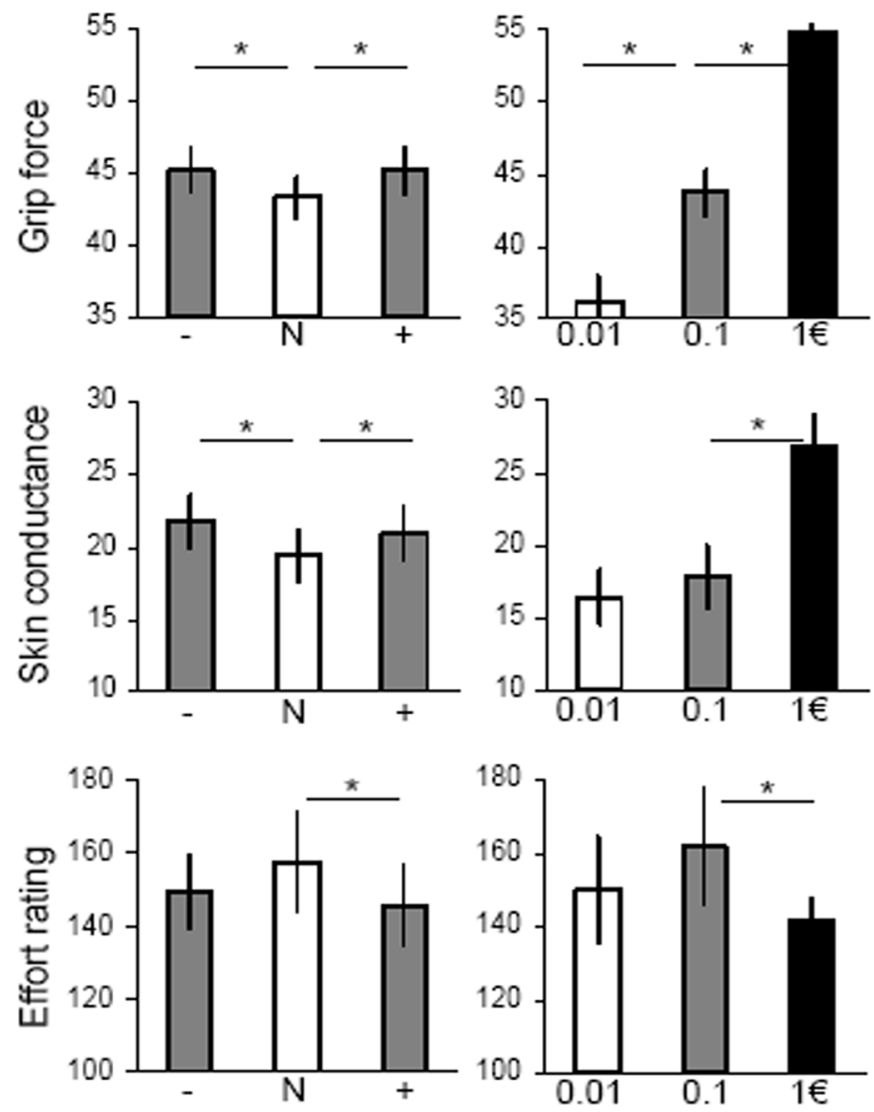

Figure 2. Behavioral data. Histograms represent mean \pm intersubject SE. $\boldsymbol{A}$, Picture rating. Emotional arousal and valence were rated on $0-10$ scales by all 60 subjects. $\boldsymbol{B}$, Emotional incentive force task. Diagrams show the main effects of emotion (induced by pictures) and motivation (induced by incentives). Grip force and skin conductance were recorded for all 60 subjects; they are expressed in proportion to the highest measure. Effort ratings were recorded in a subgroup of 20 subjects; they were normalized by the force actually produced. * Significant difference (one-tailed paired $t$ test, $p<0.05$ ). Emotional categories: + is positive, $\mathrm{N}$ is neutral, - is negative.

tive force (Fig. $2 B$, bottom). We found that effort was judged as greater in the neutral compared with the positive condition $\left(t_{(19)}\right.$ $=2.5, p<0.05)$, with a trend in that direction for the neutral versus the negative condition $\left(t_{(19)}=1.5, p=0.07\right)$. Effort was also judged as lesser in the $1 €$ condition compared with the lower incentives $\left(t_{(19)}=1.8, p<0.05\right)$. Thus, when aroused or motivated, subjects did not feel that they had to try as hard as in neutral conditions, to produce the same force. In other words, physical effort is facilitated by both emotional arousal and incentive motivation. This suggests the existence of neural pathways in the brain that are able, in situations of high arousal or motivation, 
to amplify the motor command while keeping constant the sensation of effort.

\section{Neuroimaging data}

To identify the neural pathways that mediate the influence of arousal and motivation on grip force, we searched for brain regions where the hemodynamic response, at the time of thermometer display onset, would correlate first with the subjective rating of picture-induced emotional arousal, then with the monetary incentive, and finally with the amount of force produced (Fig. 3A). Emotional arousal specifically correlated with bilateral activation of the VLPFC, centered on Broadmann's area 47 , extending into the anterior insular and temporal cortex on the left (Fig. 3A, left). Monetary incentives were associated with activity in a large BF region peaking in the ventral pallidum and encompassing the ventral striatum, extended amygdala and basal nucleus of Meynert (Fig. 3A, middle). The same cluster partially overlapped with posterior thalamic and subthalamic nuclei. In addition to this cluster, we observed bilateral activation of the insula, right activation of the cerebellum, and left activation of the primary and supplementary motor cortices. Neural correlates of force production were principally found in the left M1, which is contralateral to the hand used to squeeze the grip (Fig. $3 A$, right). Note that all main activations (VLPFC, BF, M1) survived a threshold $p<$ 0.001 after correction for multiple comparisons at the cluster level. To disclose activation below the statistical threshold (Fig. $3 B$ ), we also illustrated the $t$ values obtained in each maximum for each parameter (arousal, incentive, force).

To further investigate the participation of BF and VLPFC activities in physical effort, we extracted regression coefficients in all arousal and incentive conditions (Fig. $2 B)$. In VLPFC, regression coefficients were significantly higher for positive and negative relative to neutral pictures $\left(t_{(19)}\right.$ $=2.3, p<0.05$ and $\left.t_{(19)}=2.4, p<0.05\right)$ but not significantly different between monetary incentives. Conversely, for BF, regression coefficients were significantly higher in the $1 €$ compared with the $0.1 €$ condition $\left(t_{(19)}=1.9, p<0.05\right)$ and in the $0.1 €$ compared with the $0.01 €$ condition $\left.t_{(19)}=2.3, p<0.05\right)$ but not significantly different between emotional categories. As with behavioral data, we found no significant interaction between arousal and motivation in BF and in VLPFC either. These results confirm the dissociation found in SPMs between neural pathways involved in emotional arousal and those involved in
A

Arousal
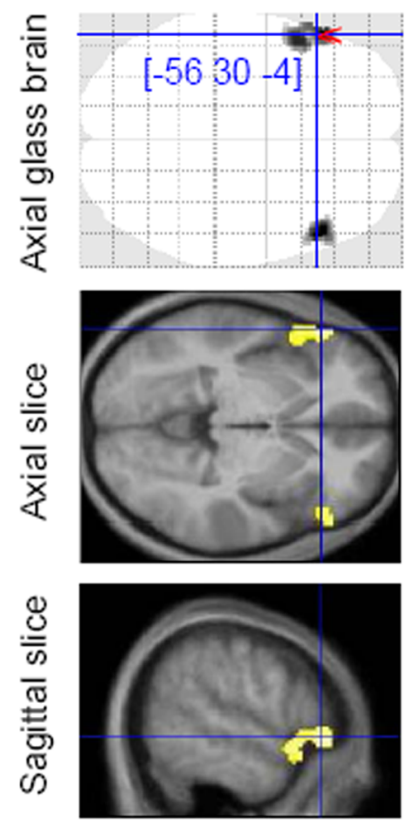

B

VLPFC

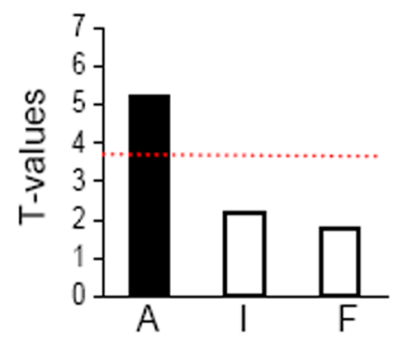

C
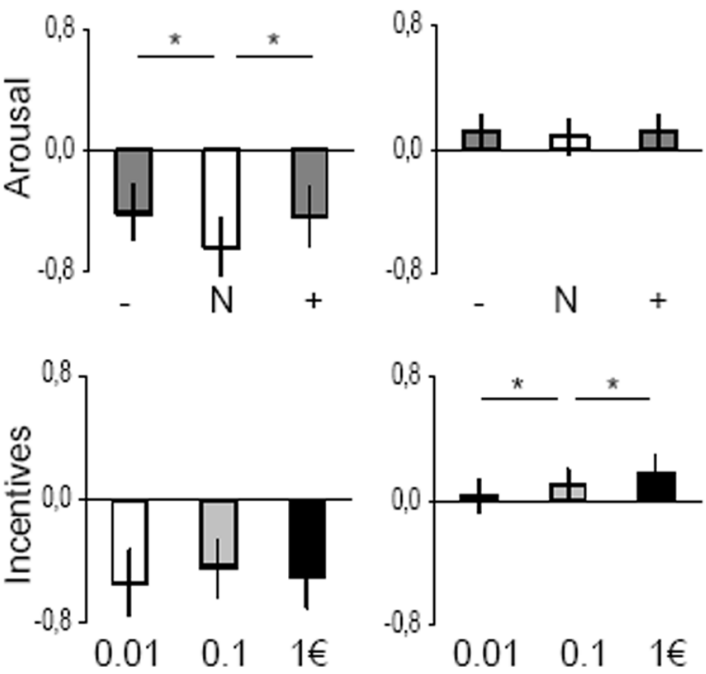

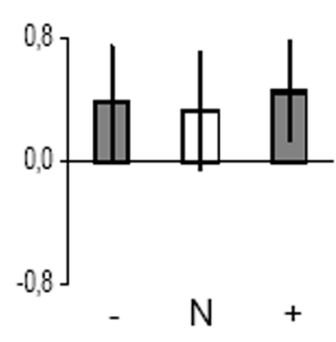

Force
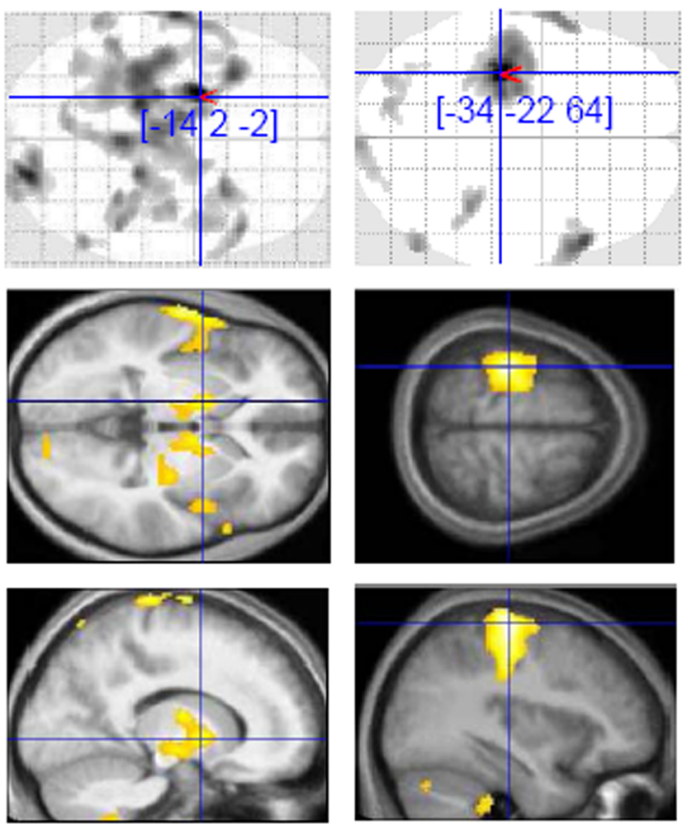

BF

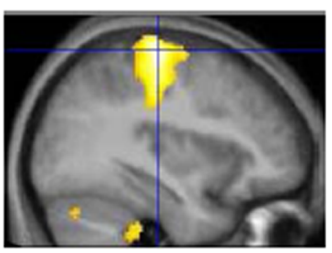

M1
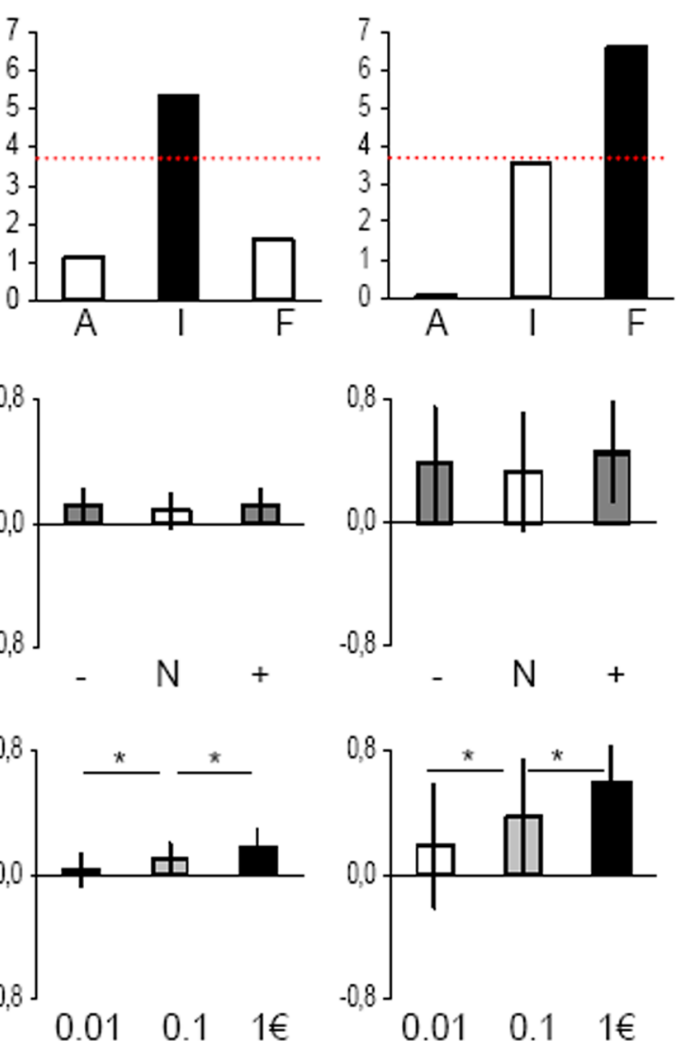

Figure 3. Neuroimaging data. $\boldsymbol{A}$, SPM of brain activity. Columns from left to right correspond to regression of the BOLD signal against arousal rating, monetary incentive, and grip force. Voxels displayed in gray on glass brain, and in yellow on slices, survived a threshold of $p<0.001$, uncorrected. The $(x, y, z)$ coordinates of maxima refer to the MNI space. Axial and sagittal slices were taken at maxima of interest indicated by red pointers on the glass brain and were superimposed on the average structural scan. $\boldsymbol{B}$, Group-level significance of the main activations. Histograms represent the $t$ value obtained in each maximum for each parameter (A, arousal; I, incentive; F, force). Dotted red lines represent a significance threshold of $p<0.001$, uncorrected. C, Categorical comparisons. Graphs show regression coefficients of the BOLD signal against the different emotional ( + is positive, $\mathrm{N}$ is neutral, - is negative) and motivational $(0.01,0.1$, and $1 €)$ categories in the same maxima as above. Histograms represent mean \pm intersubject SE. * Significant difference (one-tailed paired $t$ test, $p<0.05$ ). 


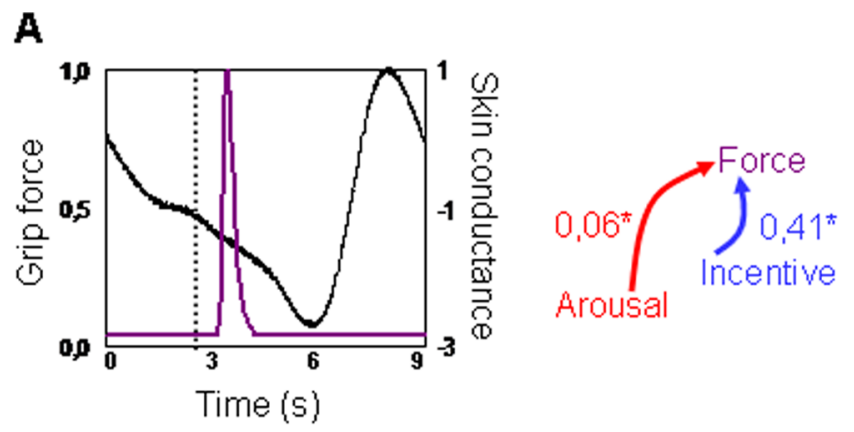

B

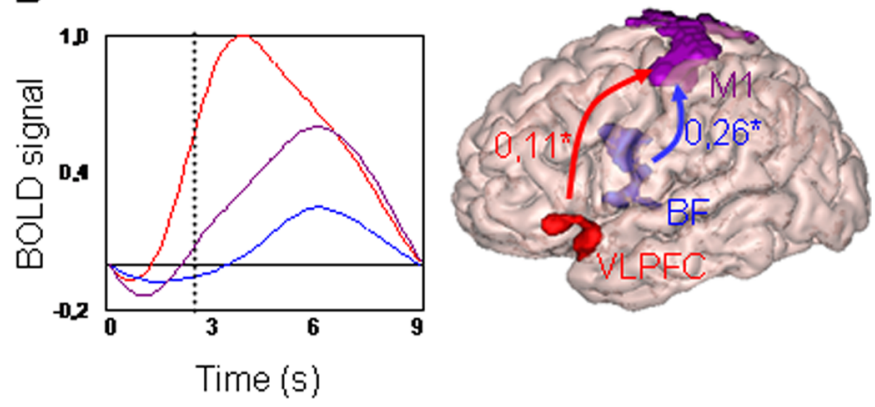

Figure 4. Relationships between main dependent measures. All variables are expressed in proportion to highest measure. $\boldsymbol{A}$, Behavioral results (all 60 subjects). Left, Time course of grip force (violet) and skin conductance (black), aligned with picture display onset (time 0) and averaged across all conditions. Right, Linear regression of force (violet) against arousal (red) and incentive (blue) variables. $\boldsymbol{B}$, Neuroimaging results (20 subjects). Left, Time curves of BOLD signal in VLPFC (red), BF (blue), and M1 (violet), aligned with picture display (time 0 ) and averaged across all conditions. Right, Linear regression of hemodynamic response in left M1 against left VLPFC and left BF. *Significant coefficients (one-tailed $t$ test, $p<0.01$ ). Vertical dotted lines in left diagrams indicate the trigger for physical effort (thermometer display onset).

incentive motivation. We also extracted the time course of the raw BOLD signal in the different maxima of interest $(\mathrm{M} 1, \mathrm{BF}$, VLPFC). Results are consistent with the VLPFC being activated by picture display and the two other regions by thermometer display (Fig. $4 B$ ). The three regions returned to baseline simultaneously at the end of trials, suggesting that VLPFC activation was prolonged all over the effort period.

Finally, we examined the relative contribution of the incentive-related $(\mathrm{BF})$ and the arousal-related (VLPFC) regions to activation of the force-related region (M1). A linear regression analysis was performed across trials on the response magnitudes, with BF and VLPFC activations as explanatory variables and M1 activation as the variable to be explained. Regression coefficients were statistically significant across subjects for both BF and $\operatorname{VLPFC}\left(r=0.26 ; t_{(19)}=7.8, p<0.001 ; r=0.11 ; t_{(19)}=3.1, p<\right.$ $0.01)$. We attempted to add as explanatory variables the interaction between BF and VLPFC, as well as random brain regions like the origin $(x=0, y=0, z=0)$ of the Montreal Neurological Institute (MNI) space: none contributed significantly. These neuroimaging results parallel those from the behavioral data: just as monetary incentives and emotional arousal significantly explained the variance in the amount of force produced on each trial, the respective underlying brain regions BF and VLPFC significantly explained the variance in M1 activation.

\section{Discussion}

Our results show that the effects of emotional arousal on force production can be dissociated from incentive motivation, at both behavioral and neuronal levels.
Behaviorally, we replicated previous findings that people, consciously or subconsciously, adapt their force according to incentive levels, whether it involves real or virtual money (Pessiglione et al., 2007; Schmidt et al., 2008). This would correspond to a trade-off between the required energy and the expected payoff. The novel finding is that emotional arousal, whatever the valence, boosts force production. This effect might help in sports requiring maximal power, such as weightlifting, where performance could thus be enhanced in highly arousing situations. It may not apply, however, to gestures requiring fine control, such as basketball free throws, since emotional arousal has been reported to impair the accuracy of movements (Noteboom et al., 2001; Coombes et al., 2007, 2008). The behavioral data also indicate that emotional arousal and incentive motivation are independent processes, since there was no significant interaction between the effect of emotional pictures and that of monetary incentives. In real life environments, the two processes may add up to improve performance, since high incentives may come in situations that are also highly arousing. In our experiment, the impact of emotional arousal on force production was much lower than that of monetary incentives, perhaps because it was incidental to the task, the explicit goal of which was to win as much money as possible. This incidental but significant effect of emotional arousal could nonetheless account for those situations in which subtle emotional boosts allow records to be broken. Furthermore, it is likely that, compared with our laboratory context, emotional arousal would be much higher for athletes in a stadium and therefore have a larger impact on behavioral energization.

The neuroimaging data revealed two different brain regions underlying emotional arousal and incentive motivation. Neuronal activity correlating with monetary incentives was principally found in basal forebrain areas (including the ventral striatum, ventral pallidum, extended amygdala, and nucleus basalis of Meynert), which have been described as forming an output system for the limbic brain, to which motivational functions are classically attributed (Heimer and Van Hoesen, 2006). The main subcluster was located in the ventral pallidum, with an anterior extension that reached the ventral striatum. This ventral striatopallidal complex, as shown by axon tracing in monkeys and fiber tracking in humans, receive inputs from the amygdala, hippocampus, orbitofrontal, and anterior cingulate cortices (Alexander et al., 1986; Haber, 2003; Lehéricy et al., 2004; Draganski et al., 2008). Consistently, it has been regularly associated with reward anticipation or receipt in functional neuroimaging studies (McClure et al., 2004; Knutson and Cooper, 2005; Peciña et al., 2006). In particular, the same regions were found to underpin incentive motivation in a previous study using the grip force paradigm (Pessiglione et al., 2007) but with a more specific activation focus. This is likely due first to the more liberal threshold that we used here, and second to the partial correlation between forces and incentives, which in our previous study had been avoided by varying the visibility of coin images.

A novel and more specific finding is the bilateral activation of the VLPFC in relation to arousal ratings of emotional pictures, which were individually recorded after the scanning session. This result suggests that VLPFC activity reflected the arousal state of the subject when about to start physical effort. Interestingly, the VLPFC has been reported to share anatomical connections with the amygdala, anterior insula, and temporal pole, which may, hence, together constitute a brain network dedicated to processing emotional information (Price, 2003; Barbas, 2007). Indeed, the VLPFC, often accompanied by the amygdala and insula, was recently shown to be activated in relation to the intensity of IAPS 
pictures, anticipation of negative pictures, negative facial expressions, affect naming, emotional distraction, reappraisal and regulation, and experienced or self-induced sadness and happiness (Lévesque et al., 2003; Pelletier et al., 2003; Habel et al., 2005; Dolcos and McCarthy, 2006; Drabant et al., 2006; Grimm et al., 2006; Lieberman et al., 2007; Mataix-Cols et al., 2008; Onoda et al., 2008; Wager et al., 2008). Abnormal activity in the same regions during emotional processing has been reported in a variety of psychiatric conditions involving emotional dysfunction such as mania, depression, negative psychotic symptoms, stress, anxiety, and obsessive compulsive disorders (Fahim et al., 2005; Monk et al., 2006; Taylor et al., 2006; Johnstone et al., 2007; Lawrence et al., 2007; Pavuluri et al., 2007; Foland et al., 2008; Guyer et al., 2008).

To be fair, we must mention that the VLPFC has also been implicated in nonemotional processing, such as attention switching or response inhibition (Swainson et al., 2003; Hampshire and Owen, 2006), although not consistently (Brody et al., 2001; Kübler et al., 2003). Consequently, one interpretation would be that the VLPFC activation reflected attentional disengagement from emotional pictures. This seems unlikely because the pictures are no longer on screen when subjects have to produce their effort. A somewhat related interpretation would be that the VLPFC underpinned inhibition or downregulation of emotional responses that may interfere with performance. However, in our case, emotional responses do not interfere with but enhance force production, such that subjects have no reason to turn them down. A more straightforward interpretation would, therefore, be that the VLPFC mediates emotional arousal and somehow drives the motor command addressed to the hand grip.

Crucially, using multiple regressions, we found that both the arousal- and the motivation-related brain regions contribute to the motor command and hence to force production. This is in line with findings that viewing an arousing picture (regardless of valence) increases motor cortex excitability (Hajcak et al., 2007) and that a multisynaptic circuit connects the basal forebrain to the primary motor cortex (Kelly and Strick, 2004). Of course, a significant regression coefficient does not prove that these regions directly drive the motor areas: some intermediate regions, possibly common to both pathways, are likely to be involved. Notably, the insula may represent a common node, since it was activated in relation to both arousal and incentive levels, as was the skin conductance response. Also, the two pathways were able to enhance the (objective) force recorded without increasing the (subjective) effort sensation. One simple interpretation would be that effort sensation arises from other brain areas, perhaps responsible for a volitional or intentional source of force production and not from arousal or motivation pathways. A slightly different interpretation would be that arousal and motivation facilitate force production because they decrease effort sensation, allowing other brain pathways to work harder without surpassing a subjective threshold of discomfort.

In conclusion, we have established, at the behavioral level, that emotional arousal can enhance force production, regardless of expected rewards and without increasing effort sensation. We also identified, at the neuronal level, part of the underlying substrate (in the ventrolateral prefrontal cortex), which is distinct from the pathways mediating influence of expected rewards. We believe that such psychological/neuronal dissociation might provide insights into psychiatric disorders such as apathy or depression. For instance, some forms of depression might involve dysfunction of the incentive motivation pathway, with potential rewards or goals failing to energize behavior. Alternatively, some variants of depression might relate to dysfunction of the second pathway, with emotional arousal paralyzing instead of energizing behavior. Dysfunction in any of these pathways might lead to patients having a higher effort sensation than healthy people while performing the same task. Thus, contrary to the common impression that these patients make no effort to improve their lot, it might be that they are already at the maximum they can withstand, even when doing seemingly easy work. Further studies are needed to assess whether our paradigm, by dissociating arousal from incentive effects on effort production, can help to better characterize depressive disorders.

\section{References}

Alexander GE, DeLong MR, Strick PL (1986) Parallel organization of functionally segregated circuits linking basal ganglia and cortex. Annu Rev Neurosci 9:357-381.

Anderson AK, Phelps EA (2001) Lesions of the human amygdala impair enhanced perception of emotionally salient events. Nature 411:305-309.

Anderson AK, Wais PE, Gabrieli JD (2006) Emotion enhances remembrance of neutral events past. Proc Natl Acad Sci U S A 103:1599-1604.

Barbas H (2007) Flow of information for emotions through temporal and orbitofrontal pathways. J Anat 211:237-249.

Brody AL, Barsom MW, Bota RG, Saxena S (2001) Prefrontal-subcortical and limbic circuit mediation of major depressive disorder. Semin Clin Neuropsychiatry 6:102-112.

Coombes SA, Cauraugh JH, Janelle CM (2007) Dissociating motivational direction and affective valence: specific emotions alter central motor processes. Psychol Sci 18:938-942.

Coombes SA, Gamble KM, Cauraugh JH, Janelle CM (2008) Emotional states alter force control during a feedback occluded motor task. Emotion 8:104-113.

Dolcos F, McCarthy G (2006) Brain systems mediating cognitive interference by emotional distraction. J Neurosci 26:2072-2079.

Dolcos F, LaBar KS, Cabeza R (2005) Remembering one year later: role of the amygdala and the medial temporal lobe memory system in retrieving emotional memories. Proc Natl Acad Sci U S A 102:2626-2631.

Drabant EM, Hariri AR, Meyer-Lindenberg A, Munoz KE, Mattay VS, Kolachana BS, Egan MF, Weinberger DR (2006) Catechol O-methyltransferase val158met genotype and neural mechanisms related to affective arousal and regulation. Arch Gen Psychiatry 63:1396-1406.

Draganski B, Kherif F, Klöppel S, Cook PA, Alexander DC, Parker GJ, Deichmann R, Ashburner J, Frackowiak RS (2008) Evidence for segregated and integrative connectivity patterns in the human basal ganglia. J Neurosci 28:7143-7152.

Fahim C, Stip E, Mancini-Marie A, Mensour B, Boulay LJ, Leroux JM, Beaudoin G, Bourgouin P, Beauregard M (2005) Brain activity during emotionally negative pictures in schizophrenia with and without flat affect: an fMRI study. Psychiatry Res 140:1-15.

Foland LC, Altshuler LL, Bookheimer SY, Eisenberger N, Townsend J, Thompson PM (2008) Evidence for deficient modulation of amygdala response by prefrontal cortex in bipolar mania. Psychiatry Res 162:27-37.

Gray JR, Braver TS, Raichle ME (2002) Integration of emotion and cognition in the lateral prefrontal cortex. Proc Natl Acad Sci USA 99:4115-4120.

Grimm S, Schmidt CF, Bermpohl F, Heinzel A, Dahlem Y, Wyss M, Hell D, Boesiger P, Boeker H, Northoff G (2006) Segregated neural representation of distinct emotion dimensions in the prefrontal cortex-an fMRI study. Neuroimage 30:325-340.

Guyer AE, Lau JY, McClure-Tone EB, Parrish J, Shiffrin ND, Reynolds RC, Chen G, Blair RJ, Leibenluft E, Fox NA, Ernst M, Pine DS, Nelson EE (2008) Amygdala and ventrolateral prefrontal cortex function during anticipated peer evaluation in pediatric social anxiety. Arch Gen Psychiatry 65:1303-1312.

Habel U, Klein M, Kellermann T, Shah NJ, Schneider F (2005) Same or different? Neural correlates of happy and sad mood in healthy males. Neuroimage 26:206-214.

Haber SN (2003) The primate basal ganglia: parallel and integrative networks. J Chem Neuroanat 26:317-330.

Hajcak G, Molnar C, George MS, Bolger K, Koola J, Nahas Z (2007) Emotion facilitates action: a transcranial magnetic stimulation study of motor cortex excitability during picture viewing. Psychophysiology 44:91-97. 
Hamann SB, Ely TD, Grafton ST, Kilts CD (1999) Amygdala activity related to enhanced memory for pleasant and aversive stimuli. Nat Neurosci 2:289-293.

Hampshire A, Owen AM (2006) Fractionating attentional control using event-related fMRI. Cereb Cortex 16:1679-1689.

Heimer L, Van Hoesen GW (2006) The limbic lobe and its output channels: implications for emotional functions and adaptive behavior. Neurosci Biobehav Rev 30:126-147.

Johnstone T, van Reekum CM, Urry HL, Kalin NH, Davidson RJ (2007) Failure to regulate: counterproductive recruitment of top-down prefrontal-subcortical circuitry in major depression. J Neurosci 27:8877-8884.

Kelly RM, Strick PL (2004) Macro-architecture of basal ganglia loops with the cerebral cortex: use of rabies virus to reveal multisynaptic circuits. Prog Brain Res 143:449-459.

Knutson B, Cooper JC (2005) Functional magnetic resonance imaging of reward prediction. Curr Opin Neurol 18:411-417.

Kübler A, Murphy K, Kaufman J, Stein EA, Garavan H (2003) Coordination within and between verbal and visuospatial working memory: network modulation and anterior frontal recruitment. Neuroimage 20:1298-1308.

Lang P, Bradley, MM, Cuthbert BN (2005) International Affective Picture System (IAPS): technical manual and affective ratings. In: National Institute of Mental Health Center for the Study of Emotion and Attention. Gainesville, FL: University of Florida.

Lawrence NS, An SK, Mataix-Cols D, Ruths F, Speckens A, Phillips ML (2007) Neural responses to facial expressions of disgust but not fear are modulated by washing symptoms in OCD. Biol Psychiatry 61:1072-1080.

Lehéricy S, Ducros M, Van de Moortele PF, Francois C, Thivard L, Poupon C, Swindale N, Ugurbil K, Kim DS (2004) Diffusion tensor fiber tracking shows distinct corticostriatal circuits in humans. Ann Neurol 55:522-529.

Lévesque J, Eugène F, Joanette Y, Paquette V, Mensour B, Beaudoin G, Leroux JM, Bourgouin P, Beauregard M (2003) Neural circuitry underlying voluntary suppression of sadness. Biol Psychiatry 53:502-510.

Lieberman MD, Eisenberger NI, Crockett MJ, Tom SM, Pfeifer JH, Way BM (2007) Putting feelings into words: affect labeling disrupts amygdala activity in response to affective stimuli. Psychol Sci 18:421-428.

Mataix-Cols D, An SK, Lawrence NS, Caseras X, Speckens A, Giampietro V, Brammer MJ, Phillips ML (2008) Individual differences in disgust sensitivity modulate neural responses to aversive/disgusting stimuli. Eur J Neurosci 27:3050-3058.

McClure SM, York MK, Montague PR (2004) The neural substrates of reward processing in humans: the modern role of FMRI. Neuroscientist 10:260-268.

Monk CS, Nelson EE, McClure EB, Mogg K, Bradley BP, Leibenluft E, Blair
RJ, Chen G, Charney DS, Ernst M, Pine DS (2006) Ventrolateral prefrontal cortex activation and attentional bias in response to angry faces in adolescents with generalized anxiety disorder. Am J Psychiatry 163:1091-1097.

Noteboom JT, Fleshner M, Enoka RM (2001) Activation of the arousal response can impair performance on a simple motor task. J Appl Physiol 91:821-831.

Ohman A, Flykt A, Esteves F (2001) Emotion drives attention: detecting the snake in the grass. J Exp Psychol Gen 130:466-478.

Onoda K, Okamoto Y, Toki S, Ueda K, Shishida K, Kinoshita A, Yoshimura S, Yamashita H, Yamawaki S (2008) Anterior cingulate cortex modulates preparatory activation during certain anticipation of negative picture. Neuropsychologia 46:102-110.

Pavuluri MN, O’Connor MM, Harral E, Sweeney JA (2007) Affective neural circuitry during facial emotion processing in pediatric bipolar disorder. Biol Psychiatry 62:158-167.

Peciña S, Smith KS, Berridge KC (2006) Hedonic hot spots in the brain. Neuroscientist 12:500-511.

Pelletier M, Bouthillier A, Lévesque J, Carrier S, Breault C, Paquette V, Mensour B, Leroux JM, Beaudoin G, Bourgouin P, Beauregard M (2003) Separate neural circuits for primary emotions? Brain activity during selfinduced sadness and happiness in professional actors. Neuroreport 14:1111-1116.

Pessiglione M, Schmidt L, Draganski B, Kalisch R, Lau H, Dolan RJ, Frith CD (2007) How the brain translates money into force: a neuroimaging study of subliminal motivation. Science 316:904-906.

Price JL (2003) Comparative aspects of amygdala connectivity. Ann N Y Acad Sci 985:50-58.

Schmidt L, d'Arc BF, Lafargue G, Galanaud D, Czernecki V, Grabli D, Schüpbach M, Hartmann A, Lévy R, Dubois B, Pessiglione M (2008) Disconnecting force from money: effects of basal ganglia damage on incentive motivation. Brain 131:1303-1310.

Swainson R, Cunnington R, Jackson GM, Rorden C, Peters AM, Morris PG, Jackson SR (2003) Cognitive control mechanisms revealed by ERP and fMRI: evidence from repeated task-switching. J Cogn Neurosci 15:785-799.

Taylor SE, Eisenberger NI, Saxbe D, Lehman BJ, Lieberman MD (2006) Neural responses to emotional stimuli are associated with childhood family stress. Biol Psychiatry 60:296-301.

Tod D, Iredale F, Gill N (2003) 'Psyching-up' and muscular force production. Sports Med 33:47-58.

Wager TD, Davidson ML, Hughes BL, Lindquist MA, Ochsner KN (2008) Prefrontal-subcortical pathways mediating successful emotion regulation. Neuron 59:1037-1050. 